\title{
Scar Symptom: Erythema and Thickness
}

Yating Yang, Xiaoli $W u$, and Wei Liu

\section{Contents}

11.1 Mechanisms of Erythema in Scar - 104

11.1.1 Inflammation-Induced Capillary Perfusion Is Crucial for Erythema Initiation - 104

11.1.2 Vascularization Dynamically Participates in the Erythema Development - 104

11.1.3 Thinner Epidermis Is Directly Responsible for the Transparency in Erythematous Scars with Skin Barrier Defect - 105

11.2 Contributions of Erythema to Scar Development and Associated Clinical Symptoms - 105

11.3 Scar Erythema and Scar Thickness - 105

11.4 Clinical Measurement of Scar Redness and Thickness - 105

11.5 Clinical Relevance - 106

11.5.1 Clinical Treatment Strategies of Erythema in Scars - 106

11.6 Clinical Treatment for Thick Scar - 107

11.7 Conclusion -107

References - 107 
Definition

Erythema is derived from the Greek erythros, which refers to the redness of skin or mucous membrane. Erythema is common in pathological scars (hypertrophic scar and keloid) and premature physiological scars where inflammation-induced angiogenesis and capillary dilation are mostly responsible. Different from the temporary and self-relieving redness in normal skin caused by allergy or mechanical friction (such as massage), the existence and severity of erythema in scar tissues are consistent with their maturity and inflammation state inside, serving as a convenient monitor of scar quality and treatment response. Scar thickness indirectly reflects the extent of scar development by collagen deposition.

\subsection{Mechanisms of Erythema in Scar}

In general, the common causes of erythema can be roughly categorized into four groups: inflammation, mechanical stimulation (massage, waxing, and tweezing of hairs), radiation (sunburn, radiotherapy), allergies and other chemical agents. Despite the pervasive nature of erythema in both physiological and pathological scars, the development mechanisms remain to be relatively less explored and therefore limited effective treatment options are available. However, previous studies demonstrated that inflammation, vascularization, and sometimes epidermis defect are the three major mechanisms that contribute to the erythema in scars.

\subsubsection{Inflammation-Induced Capillary Perfusion Is Crucial for Erythema Initiation}

Accumulated studies have confirmed that the inflammation induced by various reasons (infection, foreign body, immune dysfunction, etc.) is greatly associated with the formation of scars, especially hypertrophic scars and keloids [1]. Stimulated by inflammatory cytokines, capillaries constrict to increase the local blood flow and vessel permeability, resulting in erythema and sometimes elevated skin temperature [2]. It was shown that significant increase of blood flow values was detected in erythematous scars when compared to healthy skin $[3,4]$. Besides, erythema (measured by colorimetry) is often interpreted as an indirect measurement of the blood flow in clinical practice as it indicates the hyperemia in superficial capillaries. Bae-Harboe et al. first introduced the term "postinflammatory erythema" (PIE) in 2013 to describe erythema often seen after the resolution of inflammatory acne or other inflammatory skin conditions [5]. To be specific, the symptom of erythema persists all along the inflammatory stage of wound healing and plays multiple roles in this process.

\subsubsection{Vascularization Dynamically Participates in the Erythema Development}

The chronic inflammatory stimulation and hyperproliferation of scar tissues deteriorate the local hypoxia and promote the secretion of angiogenic factors, such as vascular endothelial growth factor (VEGF), basic fibroblast growth factor (BFGF), and hypoxia-induced factor 1 (HIF-1) [2, 6]. As reported by Amadeu et al., hypertrophic scars contain an increased number of dilated vessels in papillary and reticular dermis compared to normal skin [7]. The increased capillary density and dilated vessel diameter then contribute to the formation of erythema, in line with the study of Fullerton, who stated that erythema is determined by the blood volume under a given area, and not by the product of erythrocyte velocity and concentration [8]. Clinically, erythema disappears on finger pressure (blanching), which also suggests that the vascular remodeling is involved.

Scar erythema, blood flow, microvessels, and redness are four features of scar that are often interchangeably used under the item "vascularization" or "vascularity." However, by directly measuring erythema with colorimetry, blood flow with laser Doppler imaging (LDI), vessels with immunohistochemistry, and subjective scale of redness with POSAS, respectively, Jaspers et al. found only significant relevance between erythema and subjective redness, but no correlations between the others. In contrast, Mermans et al. found that erythema was associated with blood flow, but this correlation was not consistent at different test moments [9]. Moreover, Kischer et al. reported that most microvessels were occluded in hypertrophic scars due to an excessive number of endothelial cells $[10,11]$. And the higher hematocrit and the enlarged vessels would then result in a more sluggish blood velocity and lower blood flow values. Taken together, it is now understood that the term "vascularity" describes a dynamic process, and the four criteria mentioned above can attribute to the redness either independently or interact with each other at different stages of scar development. These exploration studies express well the complexity of erythema formation and enlighten us to view this process dynamically instead of simply putting them under the umbrella of "vascularization," especially in clinical practice. 


\subsubsection{Thinner Epidermis Is Directly Responsible for the Transparency in Erythematous Scars with Skin Barrier Defect}

The epidermal thickness and collagen synthesis are directly associated with the transparency of skin. Oliveira et al. and Busch et al. identified a thinned-out skin texture in burn scar that makes the intense circulation of blood becoming more apparent $[3,12]$. They argued that increased skin redness is not only considered as an inflammatory symptom but also the structural modification of subdermal components. And the induction of percutaneous collagen (IPC) and angiogenesis therapies (medical needling and fractional laser) has been proven to be effective to adjust the skin color after repetitive treatments [12].

Different from the thinner epidermis in physiological scars resulting from normal wound-healing process, keloids and hypertrophic scars are characterized by extra collagen deposition and increased epidermal thickness [13]. But recent studies also hypothesized a skin barrier defect in keloid scars, which may possibly alter the opacity of epidermis [14].

\subsection{Contributions of Erythema to Scar Development and Associated Clinical Symptoms}

The wound-healing process contains three chronological but partially overlapped phases: inflammatory stage (day 1-6), proliferation stage (day 4-week 3), and remodeling stage (week 3-1 year). Parallel with this process, Van Der Wal et al. reported that the erythema index scores of scars reduce significantly within 12 months [15] as a result of the common pathological mechanisms they shared. To be specific, the alleviation and deterioration of erythema are closely associated with the scar development since they indirectly reflect the inflammatory state and angiogenic activity beneath the skin. And since the redness of erythema (from pink to scarlet to purple) is the result of different proportion of hemoglobin (the red oxygenated state, absorbed at $660 \mathrm{~nm}$ ) and deoxyhemoglobin (dark red, absorbed at $940 \mathrm{~nm}$ ) in the capillaries of the dermis, the color of scarred skin can serve as a monitor of local hypoxia, angiogenesis, and microcirculation.

Although the most commonly used principles for measuring scar color are narrow-band reflectance spectrophotometry and tristimulus reflectance colorimetry
[4], researchers found statistically significant correlation between erythema values (colorimetry) and subjective redness assessment (Patient and Observer Scar Assessment Scale, POSAS) $(r=0.403, p=0.030)$ [4], proving the reliability of subjective erythema measurement in clinical practice.

Similarly, erythematous scars are more likely to suffer from pain or pruritus as a result of inflammatory stimulation and suspicious vulnerability of scar fibroblasts (previous studies detected an elevation of $\alpha$-adrenoreceptors expression on keloid fibroblasts and predicted that it might be related to paresthesia in scar tissues [16]).

Elevated temperature is directly associated with overperfusion of vessels especially arteries in deeper layer of the skin [4] and is not common in thickened scar tissues, but the existence of which could be explained as a sign of infection or active inflammatory state.

Generally, erythema reflects the inflammation and angiogenesis activity, as well as structural remodeling of scar tissues to serve as a monitor of scar maturation and quality. Since patients and clinicians often determine the success of treatment by the visual appearance of a scar, erythema measured by POSAS could be an accessible method to evaluate the treatment response and help to set the endpoint for the course.

\subsection{Scar Erythema and Scar Thickness}

There is no direct relation between scar erythema and scar thickness, because the former reflects the level of inflammation and angiogenesis process, whereas the latter indicates the extent of cell proliferation and extracellular matrix production and deposition. Such phenomenon has been frequently observed in clinical practice that a highly inflamed scar with sever erythema might be thin and soft in texture, whereas a dark-colored keloid could be thick and abundant with collagen. However, enhanced erythema by strong angiogenesis provides nutrition for fibroblast proliferation and matrix production, and therefore reducing erythema also helps to prevent scar thickening.

\subsection{Clinical Measurement of Scar Redness and Thickness}

Colorimetric plate along with photography is the conventional methodology to semi-objectively measure the redness of scars. Doppler ultrasound can detect blood flow inside a scar, but it reflects activity in a relatively deep blood vessel. For superficial blood flow inside the 
surface capillaries, laser speckle might be the technique to quantitatively measure the erythema change.

Manual measurement is the conventional way to assess scar thickness. With advances in technology, ultrasound and nuclear magnetic resonance can quantitatively provide data of scar thickness. In addition, 3D scanner not only provides the gross view of a thick scar, but also provides quantitative data of scar thickness and volume.

\subsection{Clinical Relevance}

\subsubsection{Clinical Treatment Strategies of Erythema in Scars}

Traditional clinical treatments of erythema in scar tissues mainly target on anti-inflammation and anti-proliferation, such as topical glucocorticoid and compression garments. The spreading applications of laser treatments and medical needling provide therapists with powerful arms that can interfere with erythema formation from various perspectives. Besides, systematic elements including imbalanced diets, stressful lifestyles, and even genetic predisposition may also be responsible.

\subsubsection{Anti-inflammation Strategies to Alleviate Erythema and Hinder Scars Development}

Inflammation is one of the major risk factors of both scar development and erythema. Since corticoid can effectively reduce the production of pro-inflammatory mediators and inhibit inflammatory processes in the dermis, the intralesional triamcinolone acetonide (TCA) injection has been widely used alone or as an adjuvant therapy to treat hypertrophic scars and keloids [17]. Besides, although the application of triamcinolone tapes is limited by its penetrating ability, it can be stuck to the scar area and release the drug all day long. Moreover, clinical studies observed a better performance of triamcinolone when combined with 5-Fluorouracil [17]. Other topical anti-inflammation treatment such as silicone gel sheeting that increases the elasticity of burn scar tissue also showed a reduction in pruritus, erythema, and scar thickness [18]. Systematic glucocorticoid application is not recommended for risks of complications such as diabetes, infections, and Cushing's syndrome.

\subsubsection{Laser Treatments to Interfere with Erythema from Multiple Perspective}

Laser therapy is one of the most widely used methods to treat erythema in scars. Ablative fractional lasers that create columns of vaporized tissue with surround- ing eschar and coagulated tissue can improve various features of burn scars including erythema. Kawecki et al. observed that $31 \%$ of hypertrophic burn scar patients had resolution of erythema with resulting normal skin tone after ablative fractional laser treatment [19]. Coagulating microvessels with the selective photothermolysis of wavelengths around $595 \mathrm{~nm}$, pulsed dye laser (PDL) can decrease inflammation and edema in erythematous scars and shows satisfactory outcomes in color repair. Similarly, Q-switched Nd:YAG lasers with a wavelength of $1064 \mathrm{~nm}$ in very short pulses are promising solutions to superficial angiogenesis of erythematous skin, especially in decreasing the vascular prominence in PIE $[17,18,20]$. Studies have demonstrated minimal side effects including atrophic scarring $(0.8 \%)$, hyperpigmentation $(1 \%)$, hypopigmentation $(2.6 \%)$, and dermatitis $(2 \%)$, among 500 patients treated with PDL [21]. Notably, although PDL and Nd:YAG have shown good results in reducing the red color of scars, skin erythema itself is one common adverse effect of laser treatment, which suggests that the laser therapy should be carefully selected and be practiced with cautions especially in patients with a history of post-treatment erythema or scar constitution.

\subsubsection{Compression Therapy}

Compression therapy (or occlusive dressings) has been widely applied to hypertrophic scar patients as a first-line agent with good results in decreasing erythema, thickness, and hardness of burn scars [22, 23]. A total of $60 \%$ of patients treated with compression devices showed $75-100 \%$ enhancement in scar condition according to previous studies [17]. The anti-scar effects of occlusive garments are thought to be related to occlusion and hydration [7, 17]. Some literature reported that this change is likely because of the inhibition of transforming growth factor (TGF)- $\beta 1$ release and ultimately decreased fibroblast activity [24].

\subsubsection{Medical Needling}

Although sounding contradictory, the bleeding process of medical needling can influence vascularization by stimulating angiogenesis in the post-wound healing cascade and improve the vital thickness of the epidermis (directly related to skin transparency) by inducing percutaneous collagen synthesis.

Based on the outcomes of objective measurements, medical needling achieves a normalization of the skin color and an adjustment to healthy skin after repetitive treatments [12], and it has clinically shown to improve the abnormal vascular proliferation that occurs in PIE. Similarly, fractional resurfacing lasers have been used to induce structural remodeling and skin regeneration. 


\subsection{Clinical Treatment for Thick Scar}

Contracture and functional disability caused by hypertrophic scars need surgical interventions. As for thick keloids, surgical therapy is also preferred. In addition, intralesional injection with 5-fluorouracil and steroid, cryotherapy, pressure therapy, and radiotherapy are all potential therapeutic options for thick scars, and these will be introduced in various chapters of this book and thus will not be the focus of this chapter.

\subsection{Conclusion}

The scar erythema is a burden for patients, as it amplifies the cosmetic problems and is associated with other unpleasant feelings such as pain and itching. Moreover, the development of erythema parallels with the dynamic process of inflammation, angiogenesis, scar formation, and thickening. Although most studies consider erythema as a concomitant byproduct following wound healing, it also promotes scar development via enhancing angiogenesis to provide scar fibroblasts essential nutrition for collagen production and deposition, and thus it has become an important therapeutic target as well. Although many issues remain to be explored such as what determines erythema presence, persistence, and disappearance, the erythema/redness itself can serve as a visible symptom reflecting treatment response and thus help both patients and therapists to set a relevant endpoint.

\section{Take-Home Messages \\ Both erythema and thickness are key characteristics of scar, which can be used to monitor scar development and therapy efficacy. Angiogenesis represented by scar redness is a key contributor to pathological scar development due to enhanced inflammation, which can also serve as an important therapeutic target.}

\section{References}

1. Ogawa R. Keloid and hypertrophic scars are the result of chronic inflammation in the reticular dermis. Int J Mol Sci. 2017;18:606.

2. Colgan SP, Campbell EL, Kominsky DJ. Hypoxia and mucosal inflammation. Annu Rev Pathol. 2016;11:77-100.

3. Oliveira GV, Chinkes D, Mitchell C, Oliveras G, Hawkins HK, Herndon DN. Objective assessment of burn scar vascularity, erythema, pliability, thickness, and planimetry. Dermatol Surg. 2005;31:48-58.

4. Jaspers M, Stekelenburg CM, Simons JM, Brouwer KM, Vlig M, van den Kerckhove E, van Zuijlen P. Assessing blood flow, microvasculature, erythema and redness in hypertrophic scars: a cross sectional study showing different features that require precise definitions. Burns. 2017;43:1044-50.

5. Bae-Harboe YS, Graber EM. Easy as pie (postinflammatory erythema). J Clin Aesthet Dermatol. 2013;6:46-7.

6. Sorg H, Tilkorn DJ, Hager S, Hauser J, Mirastschijski U. Skin wound healing: an update on the current knowledge and concepts. Eur Surg Res. 2017;58:81-94.

7. Amadeu T, Braune A, Mandarim-de-Lacerda C, Porto LC, Desmouliere A, Costa A. Vascularization pattern in hypertrophic scars and keloids: a stereological analysis. Pathol Res Pract. 2003;199:469-73.

8. Fullerton A, Fischer T, Lahti A, Wilhelm KP, Takiwaki H, Serup J. Guidelines for measurement of skin colour and erythema. A report from the standardization group of the european society of contact dermatitis. Contact Dermatitis. 1996;35: $1-10$.

9. Mermans JF, Peeters WJ, Dikmans R, Serroyen J, van der Hulst RR, Van den Kerckhove E. A comparative study of colour and perfusion between two different post surgical scars. Do the laser doppler imager and the colorimeter measure the same features of a scar? Skin Res Technol. 2013;19:107-14.

10. Kischer CW, Thies AC, Chvapil M. Perivascular myofibroblasts and microvascular occlusion in hypertrophic scars and keloids. Hum Pathol. 1982;13:819-24.

11. Kischer CW, Shetlar MR. Microvasculature in hypertrophic scars and the effects of pressure. J Trauma. 1979;19:757-64.

12. Busch KH, Aliu A, Walezko N, Aust M. Medical needling: effect on skin erythema of hypertrophic burn scars. Cureus. 2018;10:e3260.

13. Limandjaja GC, van den Broek LJ, Waaijman T, van Veen HA, Everts V, Monstrey S, Scheper RJ, Niessen FB, Gibbs $\mathrm{S}$. Increased epidermal thickness and abnormal epidermal differentiation in keloid scars. Br J Dermatol. 2017;176:116-26.

14. Limandjaja GC, Belien JM, Scheper RJ, Niessen FB, Gibbs S. Hypertrophic and keloid scars fail to progress from the cd34(-) /alpha-smooth muscle actin (alpha-sma)(+) immature scar phenotype and show gradient differences in alpha-sma and p16 expression. Br J Dermatol. 2020;82(4):974-86. https://doi. org/10.1111/bjd.18219.

15. van der Wal MB, Vloemans JF, Tuinebreijer WE, van de Ven $P$, van Unen E, van Zuijlen PP, Middelkoop E. Outcome after burns: an observational study on burn scar maturation and predictors for severe scarring. Wound Repair Regen. 2012;20:676-87.

16. Drummond PD, Drummond ES, Dawson LF, Mitchell V, Finch PM, Vaughan CW, Phillips JK. Upregulation of alpha1adrenoceptors on cutaneous nerve fibres after partial sciatic nerve ligation and in complex regional pain syndrome type ii. Pain. 2014;155:606-16.

17. Berman B, Maderal A, Raphael B. Keloids and hypertrophic scars: pathophysiology, classification, and treatment. Dermatol Surg. 2017;43(Suppl 1):S3-S18.

18. Sadick NS, Cardona A. Laser treatment for facial acne scars: a review. J Cosmet Laser Ther. 2018;20:424-35.

19. Kawecki M, Bernad-Wisniewska T, Sakiel S, Nowak MAndriessen A. Laser in the treatment of hypertrophic burn scars. Int Wound J. 2008;5:87-97.

20. Anderson RR, Farinelli W, Laubach H, Manstein D, Yaroslavsky AN, Gubeli J 3rd, Dylla HF. Selective photothermolysis of lipid-rich tissues: a free electron laser study. Lasers Surg Med. 2006;38:913-9.

21. Levine VJ, Geronemus RG. Adverse effects associated with the 577- and 585-nanometer pulsed dye laser in the treatment of cutaneous vascular lesions: a study of 500 patients. J Am Acad Dermatol. 1995;32:613-7. 
22. Friedstat JS, Hultman CS. Hypertrophic burn scar management: what does the evidence show? A systematic review of randomized controlled trials. Ann Plast Surg. 2014;72:S198-201.

23. Anthonissen M, Daly D, Janssens T, Van den Kerckhove E. The effects of conservative treatments on burn scars: a systematic review. Burns. 2016;42:508-18.
24. Atiyeh BS, El Khatib A, MDibo SA. Pressure garment therapy (pgt) of burn scars: evidence-based efficacy. Ann Burns Fire Disasters. 2013;26:205-12.

Open Access This chapter is licensed under the terms of the Creative Commons Attribution 4.0 International License (http://creativecommons. org/licenses/by/4.0/), which permits use, sharing, adaptation, distribution and reproduction in any medium or format, as long as you give appropriate credit to the original author(s) and the source, provide a link to the Creative Commons license and indicate if changes were made.

The images or other third party material in this chapter are included in the chapter's Creative Commons license, unless indicated otherwise in a credit line to the material. If material is not included in the chapter's Creative Commons license and your intended use is not permitted by statutory regulation or exceeds the permitted use, you will need to obtain permission directly from the copyright holder. 